\title{
THE
}

\section{Precessing Domains and the Castaing Instability in Confined Alkali-Metal Gases}

\author{
A. Kuklov
}

A. E. Meyerovich

University of Rhode Island, sfo101@uri.edu

Follow this and additional works at: https://digitalcommons.uri.edu/phys_facpubs

Terms of Use

All rights reserved under copyright.

\section{Citation/Publisher Attribution}

Kuklov, A., \& Meyerovich, A. E. (2002). Precessing Domains and the Castaing Instability in Confined Alkalimetal Gases. Phys. Rev. A, 66(2), 023607. doi: 0.1103/PhysRevA.66.023607

Available at: http://dx.doi.org/10.1103/PhysRevA.66.023607

This Article is brought to you for free and open access by the Physics at DigitalCommons@URI. It has been accepted for inclusion in Physics Faculty Publications by an authorized administrator of DigitalCommons@URI. For more information, please contact digitalcommons-group@uri.edu. 


\title{
Precessing domains and the Castaing instability in confined alkali-metal gases
}

\author{
A. Kuklov \\ Department of Physics, CUNY - Staten Island, New York, New York 10314 \\ A. E. Meyerovich \\ Department of Physics, University of Rhode Island, Kingston, Rhode Island 02881
}

(Received 11 February 2002; published 20 August 2002)

\begin{abstract}
We explore the analogy between two-component quantum alkali-metal gases and spin-polarized helium systems. Recent experiments in trapped gases are put into the frame of the existing theory for the Castaing instability in transverse channels and the formation of homogeneous precessing domains in spin-polarized systems. Analogous effects have already been observed in spin-polarized ${ }^{3} \mathrm{He}$ and ${ }^{3} \mathrm{He}-{ }^{4} \mathrm{He}$ mixture systems. The threshold effect of the confining potential on the instability is analyzed. Additional experimental possibilities for the observation of a transverse instability in a trap are discussed.
\end{abstract}

DOI: $10.1103 /$ PhysRevA.66.023607

PACS number(s): 03.75.Fi, 05.30.Jp, 51.10.+y, 75.30.Ds

\section{INTRODUCTION}

The remarkable observation of spontaneous spatial separation of states in recent experiments on trapped ${ }^{87} \mathrm{Rb}[1]$ was immediately followed by three, almost simultaneous theoretical explanations [2-4]. Though these explanations differ in details, all three exploit the analogy between a twocomponent ${ }^{87} \mathrm{Rb}$ gas and a spin-polarized spin- $\frac{1}{2}$ gas. The analogy is based on the similarity of strong molecular fields in all two-component quantum gases in the Boltzmann temperature range. In spin-polarized gases, this molecular field can cause transverse spin currents [5], which, in turn, can result in the spatial separation of the spin-up and spin-down components. An analog of this effect was, according to Refs. [2-4], observed in an experiment [1] with ${ }^{87} \mathrm{Rb}$.

The aim of this paper is to point out that the analogy between multicomponent and spin-polarized gases is even richer. In mid 1990s, there were several interesting experimental and theoretical publications on spin-polarized systems that are similar in spirit to Refs. [1-4]. The key is the so-called Castaing instability of systems with inhomogeneous polarization with respect to transverse fluctuations and the formation of precessing magnetic domains. These effects have been studied, both experimentally and theoretically, in the context of spin-polarized helium systems. We will analyze the analogs of these effects for alkali-metal gases and make suggestions for additional types of experiments including the observation of instabilities. Though the twocomponent alkali-metal gases are to a large extent similar to spin-polarized helium, the characteristic parameters are different and the issue should be revisited in the application, for example, to ${ }^{87} \mathrm{Rb}$. The main difference is the small size and nonuniformity of confined clouds of alkali-metal gases. So far, there have been no such instability experiments for alkali-metal gases though other types of dynamic instabilities have been a focus of active theoretical and experimental work in different contexts, especially for the vortex nucleation in the condensate phase (see Ref. [6], and references therein).

\section{MAIN EQUATIONS}

The analogy between spin-polarized quantum gases and multicomponent gases (gases of particles with discrete internal degrees of freedom) has been discussed in detail in Ref. [7]. For example, the densities of the components of a twocomponent gas (e.g., $\left.{ }^{87} \mathrm{Rb}\right)$ play the role of the spin-up and spin-down components of the spin-polarized gas, while the (Rabi) transitions between the states are similar to the transitions between the spin components and lead to the formation of the transverse (to the quantization axis) magnetization and correspond to the off-diagonal components of the spindensity matrix.

First, let us translate the equations of motion of Ref. [7] into the language of the trapped two-component quantum Boltzmann gas in the $s$-wave approximation (in experimentally interesting case, the gas temperature is so low that the particle wavelength is much larger than the scattering length a). For a two-component gas, the density $\hat{n}$ is a $2 \times 2$ matrix. The diagonal components $n_{1,2}$ of this matrix represent the densities of pure components and obey the conservation law

$$
\partial_{t} n_{1,2}(\mathbf{r}, t)+\nabla_{i} J_{i}^{(1,2)}(\mathbf{r}, t)=0
$$

where $J_{i}^{(1,2)}$ is the corresponding current. The off-diagonal (mixed) matrix element $n_{12}$ describes transitions between the components and obeys the equation

$$
\partial_{t} n_{12}(\mathbf{r}, t)-i \delta U(\mathbf{r}) n_{12}(\mathbf{r}, t)+\nabla_{i} J_{i}^{(12)}(\mathbf{r}, t)=I_{12}^{\text {coll }},
$$

where

$$
\delta U=U_{1}(\mathbf{r})-U_{2}(\mathbf{r})+\left(g_{11}-g_{22}\right) n(\mathbf{r}),
$$

$U_{1,2}$ is the trapping potential for the component $(1,2)$, and the interaction constants $g_{\alpha \beta}$ in ${ }^{87} \mathrm{Rb}$ are expressed via the corresponding scattering lengths as

$$
g_{\alpha \beta}=4 \pi a_{\alpha \beta} / m, \quad a_{11}=a+a^{\prime}, \quad a_{22}=a-a^{\prime}, \quad a_{12}=a,
$$

where we choose units in which $\hbar=1, m$ is the mass of a particle, and the Greek indices $\{\alpha, \beta\}$ stand for the pseu- 
dospin components $\{1,2\}$. Since the asymmetry of scattering is weak, $a^{\prime} \ll a$, the collision integral $I_{12}^{\text {coll }} \sim-\left(a^{\prime} / a\right)^{2} n_{12} / \tau$, is negligible [8] ( $\tau$ is the collision relaxation time). It is convenient to use, instead of the matrix equations, the scalar and vector pseudospin components of the densities and currents as

$$
n=n_{1}+n_{2}, \quad \mathbf{M}=\frac{1}{2}\left(n_{12}+n_{21}, i n_{21}-i n_{12}, n_{1}-n_{2}\right)
$$

and the same for the currents $J_{i}$ and $\mathbf{J}_{i}$, respectively. In these pseudospin notations, the "magnetic field" or "Larmor frequency" has the form

$$
\mathbf{\Omega}_{L}=(0,0, \delta U) .
$$

In ${ }^{87} \mathrm{Rb}$, in contrast to experiments with true spins, it is almost impossible to change the direction of the magnetic field without exciting the Rabi transitions by rf pulses. On the other hand, in helium systems the difference $U_{1}-U_{2}$ has the physical meaning of the external magnetic field while the fields $U_{1}$ and $U_{2}$ taken separately are meaningless.

In the hydrodynamic limit of small gradients, equations of motion (1)-(3) reduce to the well-known Leggett equation for the magnetic moment $\mathbf{M}(t, \mathbf{r})$ of the spin-polarized gas (see, e.g., Refs. [7,9] and references therein),

$$
\begin{gathered}
\partial_{t} \mathbf{M}+\boldsymbol{\Omega}_{L} \times \mathbf{M}+\nabla_{k} \mathbf{J}_{k}=\mathbf{0}, \\
\partial_{t} \mathbf{J}_{k}+\boldsymbol{\Omega}_{L} \times \mathbf{J}_{k}+2 g \mathbf{M} \times \mathbf{J}_{k}+(T / m) \mathbf{j}_{k}=-\mathbf{J}_{k} / \tau,
\end{gathered}
$$

where

$$
\mathbf{j}_{k}=\nabla_{k} \mathbf{M}+\mathbf{M} \nabla_{k} U / T+(n / 4 T) \nabla_{k} \boldsymbol{\Omega}_{L},
$$

$U=(1 / 2)\left(U_{1}+U_{2}+3 g n\right), \quad g=4 \pi a / m$, the symmetrized scattering length $a=\left(a_{11}+a_{22}\right) / 2$ is introduced by Eq. (4), $T$ is the temperature (here and below, the Boltzmann constant $k_{B}=1$ ), and $\tau$ is the relaxation time (in this case, the difference between the transverse and longitudinal relaxation times is vanishingly small). In equilibrium, when the currents are absent, $\mathbf{j}_{k}=\mathbf{0}$, and the "polarization" $\mathbf{M}_{0}$ follows the field and is directed along it,

$$
\mathbf{j}_{k}^{(0)} \equiv \nabla_{k} \mathbf{M}_{0}+\left[\mathbf{M}_{0} \nabla_{k} U+n_{0} \nabla_{k} \boldsymbol{\Omega}_{L} / 4\right] / T=\mathbf{0} .
$$

In Eqs. (7)-(9), the Larmor frequency can contain weak coordinate dependence, $\boldsymbol{\Omega}_{L}=\boldsymbol{\Omega}_{0}+\delta \boldsymbol{\Omega}_{L}(\mathbf{r})$. In ${ }^{87} \mathrm{Rb}$ this nonuniform part is small, $\delta \Omega_{L} / T \ll 1$. As usual, the fast rotation with Larmor frequency, $\partial_{t} \mathbf{J}_{k}+\boldsymbol{\Omega}_{0} \times \mathbf{J}_{k} \simeq \mathbf{0}$, can be accounted for by choosing the rotating reference frame in which the spin current, Eq. (8), contains only the gradient terms,

$$
\begin{gathered}
\mathbf{J}_{k}=-\frac{D}{1+\mu^{2} M_{1}^{2}}\left[\mathbf{j}_{k}+\mu \mathbf{j}_{k} \times \mathbf{M}_{1}+\mu^{2} \mathbf{M}_{1}\left(\mathbf{M}_{1} \cdot \mathbf{j}_{k}\right)\right], \\
\mu=2 g \tau, \quad \mathbf{M}_{1}=\mathbf{M}+\delta \boldsymbol{\Omega}_{L} / 2 g,
\end{gathered}
$$

where $D=\tau T / m$ is the spin diffusion coefficient. Note, that $\mu M$ is the main parameter of the theory of spin dynamics in spin-polarized quantum gases and gives the quality factor of spin oscillations.

If the magnetic field and trapping potential are uniform, $\delta \boldsymbol{\Omega}_{L}=\mathbf{0}, \nabla_{k} U=\mathbf{0}$, Eq. (11) is exactly the same as the standard Leggett equation for the spin current.

\section{FORMATION OF DOMAINS}

Often, the magnetization in polarized helium can be nonuniform and noticeably different from its equilibrium fielddriven value. The nonequilibrium polarization can be created by several techniques such as by flipping the magnetization by $180^{\circ}$ by a proper rf pulse in a part of a cell, by optical pumping, by connecting two cells with different magnetizations, etc. This initially nonuniform and nonequilibrium polarization can rapidly evolve, by means of nondissipative coherent spin currents, into a quasiequilibrium state, which will then slowly relax towards the real equilibrium. Such a quasiequilibrium state has been thoroughly studied theoretically and experimentally by Dmitriev and Fomin [10]. They demonstrated that a large initial nonequilibrium polarization $\mathbf{M}(\mathbf{r})$ can lead to the coherent formation of longitudinal magnetization domains with spatially separated up and down spins. The effect was observed in low temperature ${ }^{3} \mathrm{He}-{ }^{4} \mathrm{He}$ mixtures and normal liquid ${ }^{3} \mathrm{He}$. The existence of such domains requires that the width of the domain wall $\lambda$, which for ${ }^{87} \mathrm{Rb}$ acquires the form

$$
\lambda^{3} \sim \frac{D}{\mu M\left|\nabla \Omega_{L}\right|},
$$

should be smaller than the system size, $\lambda \ll L$. Diffusive dissipative processes lead eventually to the homogenization of the system and to the disappearance of domains. A numerical analysis of the domain formation can be found also in Ref. [11]. Note, that the field gradient $\nabla \Omega_{L}$ is crucial for maintaining the quasistationary domain state; without this gradient, a domainlike distribution of magnetization becomes unstable with respect to transverse perturbations (see the following section).

Though the main equations for spin-polarized helium and two-component alkali-metal gases are similar, there are some differences in the domain formation between helium and JILA experiments. In the JILA experiment [1], the large transverse magnetization $M_{t r} \sim n / 2$ in initial state was created by a $\pi / 2 \mathrm{rf}$ pulse. The evolution of this state led to the transient spatial separation of the pseudospin components. The final homogenization occurred on typical diffusion times. In contrast to this, in helium experiments and calculations of Ref. [10], the initial state is longitudinal. However, since the formation of domains in Ref. [10] goes through large transverse currents, the quasiequilibrium domain state is the same. A more important difference is that the experiments on alkali-metal gases are performed only in small-size traps. Taking into account the trap profile $U(x) \simeq m \omega_{0}^{2} x^{2} / 2$ and that $\mu=2 g \tau, D=T \tau / m, \delta \Omega_{L}=m \nu^{2} x^{2} / 2$, we get that in the trap of size $x \sim L=\sqrt{T / m \omega_{0}^{2}}$, the width of the domain wall is 


$$
\frac{\lambda}{L} \simeq\left[\frac{1}{4 \pi a n L^{2}} \frac{\omega_{0}^{2}}{\nu^{2}}\right]^{1 / 3}
$$

The typical values of the parameters, $\tau \approx 10^{-2} \mathrm{~s}, n \approx$ $4 \times 10^{13} \mathrm{~cm}^{-3}, \quad a=5 \times 10^{-7} \mathrm{~cm}, \quad m=1.5 \times 10^{-22} \mathrm{~g}, \quad \omega_{0}$ $\sim 7 \mathrm{~Hz}$, combined with an estimate $\nu \lesssim 0.1 \mathrm{~Hz}$, lead to

$$
\lambda / L \gtrsim 0.34 \text {. }
$$

Therefore, it is conceivable that the separation of components in the JILA experiment is similar to the formation of three Fomin-Dmitriev domains. This picture of domain formation is consistent with the results of Refs. [2-4]. The temporal symmetry of the formation and disappearance of the domains is explained by the fact that in JILA experiments $\mu M$ is not very large and the coherent processes, which are responsible for the formation of the domains, have approximately the same time constants as diffusion, which is responsible for the homogenization. Note that because of the peculiar initial condition-large transverse polarization-the formation of domains does not require the instability in the transverse channel as it is often necessary when starting from a nonequilibrium longitudinal configuration (see below).

There is another, earlier unexplained experimental fact. In the JILA experiment, the effective pseudofield gradient $\nu$ could be manipulated by changing the "real" magnetic field in the trap. It was observed [1] that the increase of this gradient $\nu$ above some threshold value, which was around $0.18 \mathrm{~Hz}$, led to some sort of unexplained stratification and/or instability. The above estimates show that at $\nu$ $\simeq 0.2 \mathrm{~Hz}$ the number of domains in this particular trap should jump to five and can give the impression of such stratification. Diffusion equilibration accelerates rapidly with an increase in the number of domains leading to the increase of gradients and decrease in the domain size. In experiment, this threshold effect can be mistaken for an instability.

\section{CASTAING-TYPE TRANSVERSE INSTABILITY: APPLICATION TO EXPERIMENTS IN TRAPS}

It is worth noting that the dynamics of the domain formation in the experiment can often start as a transverse instability in accordance with Castaing's mechanism [12]. Let us, first, briefly describe the Castaing instability in spinpolarized helium systems and then look at the implications for the JILA-type experiments.

Suppose the system is homogeneously polarized with uniform magnetization $\mathbf{M}_{0}$. If one is interested in small fluctuations $\delta \mathbf{M}$ in the transverse channel that are perpendicular to the equilibrium magnetization $\mathbf{M}_{0}$, the linearized Eqs. (7) and (11) describe the spectrum of the circularly polarized Silin spin waves in spin-polarized quantum gases (see, e.g., Ref. [9], and references therein),

$$
\omega=\Omega_{0}+\frac{D k^{2}}{1+\mu^{2} M_{0}^{2}}\left(i-\mu M_{0}\right) .
$$

When the magnetic moment is driven from the equilibrium and acquires a gradient $\nabla M_{0}$ without change in direc- tion, it is often assumed that this inhomogeneity will slowly disappear as a result of some relaxation process. This is not always the case. Often, the magnetic moment develops spontaneously a noticeable transverse component, which, in turn, may cause large longitudinal spin currents. Final relaxation towards the equilibrium is much slower. Castaing [12] showed that a purely longitudinal state with a nonequilibrium gradient of magnetization becomes unstable with respect to transverse fluctuations $\delta \mathbf{M}$. The instability occurs with respect to long-wave transverse fluctuations with the wave vectors $k^{2}<2 \mu \mathbf{k} \cdot \nabla M_{0}$. Mathematically this means that the imaginary component of the spectrum (15) acquires, according to Eq. (11), the additional term

$$
\operatorname{Im} \omega=\frac{D}{1+\mu^{2} M_{0}^{2}}\left(k^{2}-2 \mu \mathbf{k} \cdot \nabla M_{0} \frac{\mu^{2} M_{0}^{2}}{1+\mu^{2} M_{0}^{2}}\right)
$$

The imaginary part of the spectrum of transverse fluctuations near the distribution $\mathbf{M}_{0}$ becomes negative when

$$
k^{2}<2 \mu \mathbf{k} \cdot \nabla M_{0} \frac{\mu^{2} M_{0}^{2}}{1+\mu^{2} M_{0}^{2}} .
$$

The change in sign of Im $\omega$ signals the instability. Since the direction of the vector $\mathbf{k}$ is not fixed, this condition can always be satisfied in an infinite system for sufficiently longwave fluctuations. In polarized quantum gases, with the exception of small polarizations, the spin rotation parameter $\mu M$ is not small, $\mu M>1$. Then the condition (17) is roughly equivalent to

$$
k^{2}<2 \mu \mathbf{k} \cdot \nabla M_{0}
$$

This type of instability in transverse channels was observed in several experiments with ${ }^{3} \mathrm{He} \uparrow$, Refs. [13-15]. Though the conditions and the setups varied, the experiments were performed for the systems in which the coherent effects exceeded the diffusion damping, $\mu M_{0}>1$. Usually, the initial condition corresponded to a large gradient of longitudinal magnetization that could be created, for example, by a $\pi$ pulse in a part of the cell. The instability started in accordance with the Castaing criterion and often led to long-lived NMR ringing.

Though the Castaing instability in a transverse channel often serves as an initial stage of the domain formation, the instability, by itself, is not necessary for the domain formation [11]. The typical domain sizes in helium systems at temperatures $T \lesssim 10 \mathrm{mK}$ were $30 \mu \mathrm{m}-1 \mathrm{~mm}[10,11]$.

The experiment with trapped ${ }^{87} \mathrm{Rb}$ [1] was performed in such a way that the Castaing instability was not displayed. First, the polarization in the initial state was already transverse, thus eliminating the need for strong transverse currents on the initial stage of the domain formation. Second, this transverse magnetization, probably, did not have large nonequilibrium gradients. However, the Castaing instability is within an easy reach and can be observed in a slightly modified experiment. 
In JILA-type experiments, the analog of the spin rotation parameter $\mu M_{0}$ is $\tau g M_{0}$. Numerically, this parameter is $\mu M_{0}=\tau g M_{0} \sim 17 \Delta$, where $\Delta=M_{0} / n$ is the degree of pseudospin polarization. For trapped alkali-metal gases, it is difficult to achieve a high degree of the pseudospin polarization using the so-called brute force technique, i.e., the effective "magnetic field" $\Omega_{L}$. On the other hand, the preparation of the gas with a high nonequilibrium polarization is even simpler than in helium and does not require elaborate techniques such as optical pumping, Castaing-Nozieres method, etc. (see review [9]). For example, one can simply start from a pure component.

The instability should be reexamined for trapped alkalimetal gases for which the confining potential $U$ imposes an additional limitation. The reason is that in a system of size $L$, the wave vectors smaller than $1 / L$ are absent and the condition (17) must be considered in conjunction with the requirement $k>1 / L$. On the other hand, the polarization gradient $\nabla M_{0}$ introduces another length scale, $L_{M} \sim M_{0} / \nabla M_{0}$, which, depending on the magnitude of the gradient and the length over which it is spread, can be larger or smaller than $L$. As a result, the instability condition in finite traps acquires the following threshold with respect to the trap size:

$$
L_{M} / L<\frac{2 \mu^{3} M_{0}^{3}}{1+\mu^{2} M_{0}^{2}} .
$$

Roughly, the range of the "dangerous" wave vectors is $1 / L$ $<k<2 \mu M_{0} / L_{M}$.

For the instability to develop, the diffusion equilibration of the gradients should not be much faster than the time $t_{C}$ at which the instability develops. The diffusion relaxation of a longitudinal gradient $\boldsymbol{\nabla} \mathbf{M}_{0}$ takes place on a time scale of the longitudinal spin diffusion. The lower limit for this time can be estimated as $t_{D} \sim L^{2} / D$. In certain situations of open systems, the nonequilibrium gradients can be supported even on much longer times. Assuming that $\mu M_{0}$ is large and $k$ is far above the threshold, the estimate of the time $t_{C} \sim 1 /|\operatorname{Im} \omega|$ from Eq. (16) yields

$$
t_{C} \sim L_{M}^{2} / D
$$

Comparison with the diffusion time shows that the instability in small traps can be observed only if

$$
L>L_{M}
$$

This is the simplest condition for the observation of the instability in finite isolated traps.

If $L \sim L_{M}$, the diffusion time is comparable to the time of development of the instability. However, since at $\mu M \gg 1$ the instability is developed at relatively large wave vectors $k$ $>1 / L$, the signs of the instability should be still clearly visible in experiment as higher harmonics in the magnetization distribution in the transverse channel, i.e., ringing similar to that observed in helium experiments, Refs. [13-15].

Above, we neglected the possibility of having gradients of magnetic field which can stabilize the nonuniform distribution of magnetization $[10,11]$. In order to observe the insta- bility in the presence of the field gradient, Eq. (21) for $L_{M}$ should be supplemented by the condition that $L_{M}$ is also much smaller than the width of the quasistationary domain wall $\lambda$, Eq. (12),

$$
L_{M} \ll \lambda \simeq\left(\frac{D}{\mu M\left|\nabla \Omega_{L}\right|}\right)^{1 / 3} \simeq L\left[\frac{1}{4 \pi a n L^{2}} \frac{\omega_{0}^{2}}{\nu^{2}}\right]^{1 / 3} .
$$

According to the estimates from the preceding section, $\lambda$ $\gtrsim L / 3$ for the field gradients $\nu \leq 0.1 \mathrm{~Hz}$. If the inequality (22) holds, the gradients of the magnetic field cannot stabilize the system and do not impose any new restrictions for the observation of the instability.

Note, that in the situations, when the nonequilibrium gradient is supported externally, i.e., when the quasisteady currents can flow, the condition (21) is not required. Under such conditions, the scale $L_{M}$ is $L_{M} \sim L$ and the instability develops, as in helium systems, as long as $\mu M_{0} \gg 1$.

There are many experimental ways for creating a nonequilibrium gradient of the polarization of the trapped gases sufficient for the observation of the Castaing instability, Eq. (21). As a result of this instability, the polarized system should acquire a rapidly growing perpendicular component, $\mathbf{M}_{t r} \perp \mathbf{M}_{0}$. The dynamics of similar processes in helium, though in very different geometries, has been studied computationally in Ref. [11].

In experiment [1], the $\pi / 2 \mathrm{rf}$ pulse created the almost uniform transverse polarization $\mathbf{M}_{0}$ in, i.e., the $x$ direction in the pseudospin space. The spin-rotation parameter was relatively large,

$$
\mu M_{0} \approx \tau g n / 2 \sim 9 .
$$

[The trapping potential was responsible for some nonuniformity of polarization. This equilibrium nonuniformity could not lead to any instability; actually, small field gradients help to stabilize the system.] The small fluctuations $\delta \mathbf{M}$ around this configuration were, if one ignores the small magnetic field, the gapless Silin waves, Eq. (15), and there was no instability. However, if the initial polarization $M_{x}$ in the experiment [1] were sufficiently nonuniform for the instability to occur, the observed component separation, i.e., the formation of domains with different $M_{z}$, would have taken place much faster than in Refs. [1-4]. This can be checked experimentally.

There are several other possible experiments with the instability and domain formation that can be performed with trapped alkali-metal gases along the lines of the helium experiments. First, one can start from a one-component gas and flip the "magnetization" by $180^{\circ}$, i.e., excite the Rabi transition, in a part of a trap. Then one can observe long-time magnetization ringing in the system similar to Refs. [13-15]. In this experiment, however, the transverse polarization might already exist after the pulse on the interface between the components due to some residual nonuniformity of the Rabi frequency [16]. In this sense, it is not clear whether the instability can be unambiguously identified.

The next experiment involves bringing together two clouds consisting of the two different components, which 
have no memory of each other. Employing the pseudospin language, these two clouds are characterized by the opposite polarizations in the $z$ direction with no transverse components. Then, the dynamics of the mixing of these two clouds can proceed along the line of the Castaing instability, as opposed to a trivial (incoherent) diffusion mixing. Accordingly, the transverse nonuniform magnetization will arise spontaneously near the interface between the mixing components. Then, it will slowly relax till the equilibrium state is reached. In some sense, this effect is the time-reversal analog of the experiment [1]. Indeed, in Ref. [1], the initial nonequilibrium transverse polarization resulted in the transient separation of the components. The effect of the Castaing instability results in the spontaneous creation of the transverse polarization in the process of the components mixing.

Another possibility is to create a nonuniform stable distribution of longitudinal polarization in which the criterion (21) is violated and then to change rapidly the size of the trap so as to comply with the instability condition (21).

The last suggestion is more speculative. The nonuniformity of the initial nonequilibrium configuration can be caused by the sensitivity of the two-photon Rabi transition to the gradients of the difference of the trapping potentials [16], that is, to the value of the Larmor frequency $\Omega_{L}$. This spacial nonuniformity of the pulse frequency $\Omega(\mathbf{r})$ results in the formation of helicoidal structures after the pulse. For example, if the Rabi rotation takes place around the $x$ axis, the structure of magnetization $\mathbf{M}(\mathbf{r})$ after the pulse of duration $t$ has the form $M_{z}+i M_{y}=M_{0} \exp [i \Omega(\mathbf{r}) t]$. Such helicoidal polarization $\mathbf{M}(\mathbf{r})$ has large gradients and may be unstable. As was emphasized in Ref. [16], the Rabi frequency gradient $\nabla \Omega$ can be controlled with high accuracy. Accordingly, one can control the magnetization gradients. However, the time evolution of the helicoidlike structure $\mathbf{M}(\mathbf{r})$ is described by a convoluted set of coupled transverse and longitudinal equations. At present, it is not clear as to what extent the instability condition for such helicoids differs from the above equations for simple geometries. This general suggestion can be modified in various ways so as to create different structures, some of which can be even one dimensional and are easier to describe theoretically than the helicoids of a general form. However, a practical implementation strongly depends on an available experimental setup.
In most of the suggested experiments, the detection of the instability and the spontaneous formation of the transverse magnetization, i.e., of the large off-diagonal components of the density matrix associated with continuous Rabi transitions, can be done using the Ramsey spectroscopy [17]. Though some of the effects discussed above exist in both hydrodynamic and semiballistic regimes, the direct application of the results requires that the size of the trap $L$ and the characteristic length $L_{M}$ should be larger than the particle mean free path $1 / 4 \pi a^{2} n$. In the typical experimental conditions this means that the sizes should exceed $100 \mu \mathrm{m}$. Another necessary condition, $\mu M>1$, is almost identical to the condition for the quantum gas regime and holds practically in all current experiments with trapped alkali-metal gases.

\section{CONCLUSIONS}

In conclusion, we pursued the analogy between the twocomponent ${ }^{87} \mathrm{Rb}$ gases and spin-polarized helium systems. The observed separation of components in experiment [1] is analogous to the formation of coherent precessing domains in helium [10]. This interpretation is consistent with the conclusions of Refs. [2-4]. The width of the domain wall agrees with the formation of up to three domains. An increase in the gradient of the pseudomagnetic field can result in a spontaneous stratification of the system. A polarization gradient in the initial state can drastically speed up the domain formation. Apart from the observation of the domain formation, there are several other interesting experimental possibilities associated with the pseudospin Castaing instability in transverse channel. This instability is almost inevitable in highly polarized systems with a nonuniform distribution of the pseudospin and can be observed in the JILA-type experimental setups. The conditions for this instability in finite traps are delineated.

\section{ACKNOWLEDGMENTS}

The authors acknowledge support by the CUNY, Grant No. PSC-63499-0032 (A.K.), and by the NSF, Grant No. DMR-0077266 (A.E.M.).
[1] H.J. Lewandowski, D.M. Harber, D.L. Whitaker, and E.A. Cornell, Phys. Rev. Lett. 88, 070403 (2002).

[2] M.Ö. Oktel and L.S. Levitov, Phys. Rev. Lett. 88, 230403 (2002).

[3] J.N. Fuchs, D.M. Gangardt, and F. Laloë, Phys. Rev. Lett. 88, 230404 (2002).

[4] J.E. Williams, T. Nikuni, and C.W. Clark, Phys. Rev. Lett. 88, 230405 (2002).

[5] C. Lhuillier and F. Laloë, J. Phys. (Paris) 43, 197 (1982); 43, 225 (1982); E.P. Bashkin and A.E. Meyerovich, Adv. Phys. 30, 1 (1981); E.P. Bashkin, Pis'ma Zh. Eksp. Teor. Fiz. 33, 11 (1981) [JETP Lett. 33, 8 (1981)]; L. Levy and A. Ruckenstein,
Phys. Rev. Lett. 52, 1512 (1984).

[6] F. Dalfovo and S. Stringari, Phys. Rev. A 63, 011601(R) (2001); K.W. Madison, F. Chevy, V. Bretin, and J. Dalibard, Phys. Rev. Lett. 86, 4443 (2001); A. Recati, F. Zambelli, and S. Stringari, ibid. 86, 377 (2001); T. Isoshima and K. Machida, Phys. Rev. A 60, 3313 (1999); S. Sinha and Y. Castin, Phys. Rev. Lett. 87, 190402 (2001); J.E. Williams, E. Zaremba, B. Jackson, T. Nikuni, and A. Griffin, ibid. 88, 070401 (2002).

[7] A.E. Meyerovich, Phys. Rev. B 39, 9318 (1989).

[8] A.B. Kuklov and J.L. Birman, Phys. Rev. Lett. 85, 5488 (2000).

[9] A.E. Meyerovich, in Prog. Low Temp. Phys., edited by D.F. Brewer (North-Holland, Amsterdam, 1987), Vol. 11, pp. 1-73; 
A.E. Meyerovich, in Helium Three, edited by W.P. Halperin and L.P. Pitaevski (Elsevier, Amsterdam, 1990), pp. 757-879.

[10] V.V. Dmitriev and I.A. Fomin, Pis'ma Zh. Eksp. Teor. Fiz. 59, 353 (1994) [JETP Lett. 59, 378 (1994)]; I.A. Fomin, J. Low Temp. Phys. 101, 749 (1995); V.V. Dmitriev, V.V. Moroz, and S.R. Zakazov, ibid. 101, 141 (1995); V.V. Dmitriev, Czech. J. Phys. 46, Suppl. S6 30113018 (1996).

[11] R.J. Ragan and D.M. Schwarz, J. Low Temp. Phys. 109, 766 (1997).

[12] B. Castaing, Physica B \& C 126, 212 (1984).

[13] H. Akimoto, O. Ishikawa, G.-H. Oh, M. Nakagawa, T. Hata, and T. Kodama, J. Low Temp. Phys. 82, 295 (1991).

[14] G. Nunes, C. Jin, D.L. Hawthorne, A.M. Putnam, and D.M. Lee, Phys. Rev. B 46, 9082 (1992).

[15] R. Konig, J.H. Ager, R.M. Bowley, J.R. Owers-Bradley, and A.E. Meyerovich, J. Low Temp. Phys. 101, 833 (1995).

[16] M.R. Matthews, B.P. Anderson, P.C. Haljan, D.S. Hall, M.J. Holland, J.E. Williams, C.E. Wieman, and E.A. Cornell, Phys. Rev. Lett. 83, 3358 (1999).

[17] D.S. Hall, M.R. Matthews, C.E. Wieman, and E.A. Cornell, Phys. Rev. Lett. 81, 1543 (1998). 